\title{
Uso de drogas por estudantes de Farmácia da Universidade Federal do Espírito Santo
}

\author{
Use of drugs by Pharmacy students at the \\ Federal University in Espírito Santo \\ Flávia Batista Portugal', Renata Santos de Souza', Vitor Buaiz', Marluce Miguel de Siqueira'
}

\section{RESUMO}

Objetivo: Traçar o perfil dos usuários de substâncias psicoativas do curso de Farmácia do Centro de Ciências da Saúde (CCS) da Universidade Federal do Espírito Santo (Ufes). Métodos: Tratou-se de estudo exploratório, descritivo, transversal e quantitativo. Foram pesquisados 148 alunos $(80,4 \%)$ matriculados no curso de Farmácia. O instrumento utilizado foi o questionário da Organização Mundial de Saúde adaptado à realidade brasileira. Os dados foram tabulados e analisados por meio do programa Statistical Packcage for the Social Science (SPSS). Resultados: Dos pesquisados, a maior representação foi na faixa etária de 20 a 22 anos (52,7\%), do sexo feminino (67,6\%), e da classe socioeconômica B (47,3\%). Quanto ao uso de álcool, o "uso na vida" foi de 87,8\%, "uso no ano" de 77,7\% e "uso no mês" de 58,1\%. Entre as outras drogas, o "uso na vida" mais prevalente foi dos inalantes (18,2\%).

\section{Palavras-chave}

Drogas, universitários, prevenção.

\section{Keywords}

Drugs, university students, prevention.
Conclusão: Conforme observado, o uso de substâncias psicoativas entre universitários é questão preocupante, demonstrando a importância de maior abordagem à temática no currículo acadêmico.

\begin{abstract}
Objective: To trace the profile of the drug use between the university students of the course of Pharmacy Sciences Health Center of Federal University of Espirito Santo. Methods: One is about explorer, descriptive, transversal and quantitative study. A survey was conducted with 148 undergraduate pharmacy students (80.4\%). The instrument used is an adaptation of the developed one for the WHO. The data had been tabulated and analyzed through the Statistical Packcage for the Social Science (SPSS). Results: $67.6 \%$ of the university students are female, $52.7 \%$ it had between 20 and 22 years and $47.3 \%$ belong to the social class B. Regarding the use of alcohol, $87.8 \%$ made use in the life, $77.7 \%$ in the year and $58.1 \%$ in the month. Between the other drugs, the use in life of inahaled drugs (18.2\%) was the most common. Conclusion: As observed, the undergraduate students belong a concerned group, showing the significance of a better approach in this subject at college's curriculum.
\end{abstract}




\section{INTRODUÇÃO}

Atualmente, a questão do uso de substâncias psicoativas está em evidência, não só pelas suas conseqüências à economia e à sociedade, mas, principalmente, às suas implicações no que diz respeito à saúde da população'. O uso de substâncias psicoativas atinge todas as camadas da sociedade e, ultimamente, seu uso está cada vez mais precoce ${ }^{2}$, atingindo cada vez mais os estudantes.

Em razão disso, há grande preocupação com o uso de substâncias psicoativas entre universitários. Apesar de poderem superar a fase de "uso pesado" e as conseqüências desse consumo sem assistência nem tratamento, eles são vulneráveis à grande quantidade de conseqüências prejudiciais até que abandonem o uso3.

O consumo de substâncias psicoativas entre universitários da área da saúde é assunto que deve receber atenção especial, pois, no cotidiano desses futuros profissionais, eles serão responsáveis pela identificação e pelo encaminhamento de pacientes com problemas relacionados ao uso de substâncias psicoativas e, também, servirão de exemplos para seus pacientes ${ }^{4}$. A necessidade de maior conhecimento sobre o uso de substâncias psicoativas entre acadêmicos de Farmácia deve-se ao fato de que esses universitários, em sua vida profissional, poderão detectar e prevenir o uso e o abuso de substâncias psicoativas, monitorando os aumentos das dosagens e a duração de uso; se mais de uma medicação está sendo utilizada com a mesma indicação; e reconhecer pacientes atendidos por dois médicos e, também, as falsas prescrições 5 .

Desse modo, o presente estudo teve como objetivo traçar o perfil do uso de substâncias psicoativas entre os universitários do curso de Farmácia do Centro de Ciências da Saúde (CCS) da Universidade Federal do Espírito Santo (Ufes).

\section{MÉTODOS}

A pesquisa foi realizada no curso de Farmácia e Bioquímica do CCS-Ufes, no período de junho a julho de 2007. A população estuda foi constituída de alunos matriculados no referido curso nos nove períodos, totalizando 184 alunos. A amostra foi calculada no software Epi Info 6.04, considerando-se o grau de confiabilidade de $95 \%$ e a precisão de $2 \%$. Desse modo, a amostra final foi constituída de 148 estudantes.

A pesquisa foi aprovada pelo Comitê de Ética em Pesquisa da Ufes, por meio do Processo no 104/2006. Inicialmente, foi enviada carta aos chefes de departamento do curso de Farmácia, solicitando a colaboração dos professores na concessão de alguns minutos de sua aula. Posteriormente, foi realizado estudo-piloto com três acadêmicos do início, meio e fim do curso de Farmácia e Bioquímica da Ufes. Para a realização da pesquisa, foi utilizado questionário fechado, de autopreenchimento e sem identificação pessoal do aluno. A aplicação do questionário foi realizada em sala de aula, por bolsistas de iniciação científica devidamente treinados, com anuência prévia dos professores, após breve explicação dos objetivos do trabalho pelos pesquisadores. Não era obrigatório o preenchimento, dando-se ao aluno a liberdade de devolvê-lo em branco, porém todos que aceitaram participar da pesquisa assinaram o termo de consentimento livre e esclarecido (TCLE), de acordo com Resolução no 196/1996. O questionário utilizado é uma adaptação do instrumento proposto pela Organização Mundial de Saúde $(\mathrm{OMS})^{6}$. No Brasil, foi adaptado por Carlini-Cotrim et al. ${ }^{2}$, sendo utilizado em levantamentos sobre substâncias psicoativas pelo Centro Brasileiro de Informações sobre Drogas Psicotrópicas (Cebrid).

O instrumento é dividido em cinco partes: a) na primeira há a explicação da pesquisa, convidando o estudante a participar; b) na segunda parte envolve questões referentes a dados sociodemográficos; $c$ ) na terceira engloba os padrões de uso não-médico de psicotrópicos (álcool, tabaco, maconha, alucinógenos, cocaína, anfetaminas, anticolinérgicos, solventes orgânicos, tranqüilizantes ansiolíticos, opiáceos, sedativos e barbitúricos), além de questões sobre anabolizantes, bem como drogas injetáveis; d) a quarta consiste de questionamento minucioso sobre o uso de álcool; e e) a quinta parte envolve questionamento sobre relacionamento familiar.

A análise dos dados foi realizada pelo programa Statistical Packcage for the Social Science (SPSS), versão 20057, seguida da análise descritiva das variáveis, de acordo com a classificação da $\mathrm{OMS}^{6}$ sobre o uso de drogas: "uso na vida" (quando a pessoa fez uso de qualquer droga psicotrópica pelo menos uma vez na vida); "uso no ano" (quando a pessoa utilizou droga psicotrópica pelo menos uma vez nos 12 meses que antecederam a pesquisa); "uso no mês" (quando a pessoa utilizou droga psicotrópica pelo menos uma vez nos 30 dias que antecederam a pesquisa); "uso freqüente" (quando a pessoa utilizou droga psicotrópica seis ou mais vezes nos 30 dias que antecederam a pesquisa); "uso pesado" (quando a pessoa utilizou droga psicotrópica 20 ou mais vezes nos 30 dias que antecederam a pesquisa).

\section{RESULTADOS}

A Tabela 1 apresenta os dados sociodemográficos dos estudantes pesquisados, mostrando predomínio da faixa etária de 20 a 22 anos (52,7\%) e prevalência do sexo feminino (67,6\%). Em relação à classe socioeconômica, houve prevalência da classe B (47,3\%), seguida das classes A $(29,1 \%)$ e C $(20,9 \%)$. 
Tabela 1. Distribuição dos estudantes do curso de Farmácia e Bioquímica da Universidade Federal do Espírito Santo, segundo suas características.

\begin{tabular}{|c|c|c|}
\hline \multirow[t]{2}{*}{ Características } & \multicolumn{2}{|c|}{ Estudantes } \\
\hline & $\mathbf{n}$ & $\%$ \\
\hline \multicolumn{3}{|l|}{ Sexo } \\
\hline Masculino & 47 & 31,8 \\
\hline Feminino & 100 & 67,6 \\
\hline Não informado & 1 & 0,7 \\
\hline Total & 148 & 100,0 \\
\hline \multicolumn{3}{|l|}{ Idade (anos) } \\
\hline $17-19$ & 32 & 21,6 \\
\hline $20-22$ & 78 & 52,7 \\
\hline $23-25$ & 29 & 19,6 \\
\hline $26-28$ & 4 & 2,7 \\
\hline $29-31$ & 1 & 0,7 \\
\hline $32-24$ & 2 & 1,4 \\
\hline Não informado & 2 & 1,4 \\
\hline Total & 148 & 100,1 \\
\hline \multicolumn{3}{|c|}{ Defasagem na universidade } \\
\hline Nenhuma falta & 64 & 43,2 \\
\hline 1 a 3 faltas & 43 & 29,1 \\
\hline 4 a 8 faltas & 22 & 14,9 \\
\hline 9 ou mais faltas & 19 & 12,8 \\
\hline Total & 148 & 100,0 \\
\hline \multicolumn{3}{|l|}{ Nível socioeconômico } \\
\hline A & 43 & 29,1 \\
\hline B & 70 & 47,3 \\
\hline$C$ & 31 & 20,9 \\
\hline D & 4 & 2,7 \\
\hline $\mathrm{E}$ & 0 & 0,0 \\
\hline Total & 148 & 100,0 \\
\hline
\end{tabular}

Os dados da Tabela 2 mostram que o uso na vida de substâncias psicoativas é maior entre as substâncias lícitas. Assim, o uso na vida de álcool correspondeu a $86,5 \%$, enquanto o de tabaco $28,4 \%$. Entre as drogas ilícitas de maior uso na vida, encontram-se os inalantes (18,2\%), os ansiolíti$\cos (9,1 \%)$, a maconha $(8,8 \%)$ e as anfetaminas $(8,1 \%)$.

Quanto ao uso no ano, as drogas lícitas foram as mais utilizadas (álcool e tabaco com $77,7 \%$ e 12,2\%, respectivamente), e entre as ilícitas, os ansiolíticos (7,4\%), a maconha (6,1\%) e as anfetaminas $(6,1 \%)$ foram as mais prevalentes. Quanto ao uso no mês, nota-se maior uso de álcool (58,1\%), tabaco (5,5\%), ansiolíticos (4,1\%), maconha (3,4\%), anfetaminas (2,7\%) e inalantes $(1,4 \%)$. Tanto no uso freqüente quanto pesado, o álcool é a substância mais prevalente, 18,2\% e 8,1\%, respectivamente.

Na Tabela 3, que demonstra o uso na vida das substâncias psicoativas por sexo, observa-se semelhança no uso de certas drogas, como álcool e anfetaminas. Dos estudantes entrevistados, 89,4\% dos homens relataram ter experimentado o álcool e $85 \%$ das mulheres também referiram o uso; para as anfetaminas, $8,5 \%$ dos homens mencionaram o uso e $8 \%$ das mulheres também. Já entre outras drogas, foi possível observar maior "uso na vida" dos homens, por exemplo, o tabaco que foi relatado por $40,4 \%$ dos homens, enquanto $22 \%$ das mulheres. Os barbitúricos e os opióides apresentaram freqüência de uso nas mulheres e nenhum relato entre os homens.

Conforme mostra a Tabela 4, a idade em que os universitários iniciaram o consumo de bebidas alcoólicas variou de 13 a 15 anos (20,9\%) e 16 a 18 anos (21,6\%). Para aqueles que
Tabela 2. Distribuição dos estudantes do curso de Farmácia e Bioquímica da Universidade Federal do Espírito Santo, segundo o uso de substâncias psicoativas.

\begin{tabular}{|c|c|c|c|c|c|c|c|c|c|c|}
\hline \multirow[t]{2}{*}{ Substância psicoativa } & \multicolumn{2}{|c|}{ Uso na vida } & \multicolumn{2}{|c|}{ Uso no ano } & \multicolumn{2}{|c|}{ Uso no mês } & \multicolumn{2}{|c|}{ Uso freqüente } & \multicolumn{2}{|c|}{ Uso pesado } \\
\hline & $\mathrm{n}$ & $\%$ & $\mathrm{n}$ & $\%$ & n & $\%$ & $\mathrm{n}$ & $\%$ & $\mathrm{n}$ & $\%$ \\
\hline Álcool & 128 & 86,5 & 115 & 77,7 & 86 & 58,1 & 27 & 18,2 & 12 & 8,1 \\
\hline Tabaco & 42 & 28,4 & 18 & 12,2 & 6 & 5,5 & 1 & 0,7 & 2 & 1,4 \\
\hline Inalantes & 27 & 18,2 & 5 & 3,4 & 2 & 1,4 & 0 & 0,0 & 0 & 0,0 \\
\hline Ansiolíticos & 14 & 9,5 & 11 & 7,4 & 6 & 4,1 & 0 & 0,0 & 0 & 0,0 \\
\hline Maconha & 13 & 8,8 & 9 & 6,1 & 5 & 3,4 & 0 & 0,0 & 1 & 0,7 \\
\hline Anfetaminas & 12 & 8,1 & 9 & 6,1 & 4 & 2,7 & 1 & 0,7 & 0 & 0,0 \\
\hline Cocaína & 2 & 1,4 & 1 & 0,7 & 0 & 0,0 & 0 & 0,0 & 0 & 0,0 \\
\hline Barbitúricos & 1 & 0,7 & 0 & 0,0 & 0 & 0,0 & 0 & 0,0 & 0 & 0,0 \\
\hline Anticolinérgicos & 1 & 0,7 & 0 & 0,0 & 0 & 0,0 & 0 & 0,0 & 0 & 0,0 \\
\hline Opióides & 1 & 0,7 & - & - & - & - & - & - & - & - \\
\hline Alucinógenos & 1 & 0,7 & - & - & - & - & - & - & - & - \\
\hline Xaropes & 1 & 0,7 & - & - & - & - & - & - & - & - \\
\hline Anabolizantes & 1 & 0,7 & - & - & - & - & - & - & - & - \\
\hline Orexígenos & 0 & 0,0 & - & - & - & - & - & - & - & - \\
\hline
\end{tabular}

Tabela 3. Distribuição dos estudantes do curso de Farmácia e Bioquímica da Universidade Federal do Espírito Santo, segundo uso na vida de drogas por sexo.

\begin{tabular}{|c|c|c|c|c|c|}
\hline \multirow[t]{2}{*}{ Drogas } & \multicolumn{2}{|c|}{ Masculino } & \multicolumn{2}{|c|}{ Feminino } & \multirow{2}{*}{$\begin{array}{c}\text { Total } \\
\mathrm{n}\end{array}$} \\
\hline & $n$ & $\%$ & $n$ & $\%$ & \\
\hline Álcool & 42 & 89,4 & 85 & 85 & 127 \\
\hline Tabaco & 19 & 40,4 & 22 & 22 & 41 \\
\hline Inalantes & 11 & 23,4 & 16 & 16 & 27 \\
\hline Ansiolíticos & 6 & 12,8 & 7 & 7 & 13 \\
\hline Maconha & 7 & 14,9 & 6 & 6 & 13 \\
\hline Anfetaminas & 4 & 8,5 & 8 & 8 & 12 \\
\hline Cocaína & 2 & 4,3 & 0 & 0 & 2 \\
\hline Barbitúricos & 0 & 0 & 1 & 1 & 1 \\
\hline Anticolinérgicos & 1 & 2,1 & 0 & 0 & 1 \\
\hline Opióides & 0 & 0 & 1 & 1 & 1 \\
\hline Alucinógenos & 1 & 2,1 & 0 & 0 & 1 \\
\hline Xaropes & 1 & 2,1 & 0 & 0 & 1 \\
\hline Anabolizantes & 1 & 2,1 & 0 & 0 & 1 \\
\hline
\end{tabular}

relataram o uso de tabaco, a faixa etária de início do uso de maior freqüência foi de 13 a 15 anos (31\%), seguida de 16 a 18 anos, 19 a 21 anos (29\%) e 10 a 12 anos (14,3\%). Dos entrevistados, apenas 1,4\% fumava de um a dez cigarros por dia e 0,7\% fumava de 11 a 20 cigarros por dia. Quanto às substâncias ilícitas, a idade de experimentação da maconha variou de 16 a 18 anos (53,8\%) e acima de 18 anos (23,1\%). Entre as anfetaminas, 33,3\% dos alunos experimentaram entre $16 \mathrm{e}$ 18 anos e a mesma porcentagem acima dos 18 anos. Dos universitários entrevistados que já fizeram uso de ansiolíticos, 57,1\% experimentaram com idade superior a 18 anos, $14,3 \%$ com 16 a 18 anos e 7,1\% entre 10 e 12 anos. 
Tabela 4. Distribuição dos estudantes do curso de Farmácia e Bioquímica da Universidade Federal do Espírito Santo, segundo a idade de início do uso.

\begin{tabular}{|c|c|c|c|c|c|c|c|c|c|c|c|c|}
\hline \multirow[t]{2}{*}{ Droga } & \multicolumn{2}{|c|}{$\begin{array}{l}\text { Abaixo de } \\
10 \text { anos }\end{array}$} & \multicolumn{2}{|c|}{$10-12$ anos } & \multicolumn{2}{|c|}{ 13-15 anos } & \multicolumn{2}{|c|}{ 16-18 anos } & \multicolumn{2}{|c|}{$\begin{array}{l}\text { Acima de } \\
18 \text { anos }\end{array}$} & \multicolumn{2}{|c|}{$\begin{array}{l}\text { Não } \\
\text { lembra }\end{array}$} \\
\hline & $\mathrm{n}$ & $\%$ & $n$ & $\%$ & $n$ & $\%$ & $n$ & $\%$ & $n$ & $\%$ & $n$ & $\%$ \\
\hline Álcool & 5 & 3,8 & 11 & 8,4 & 31 & 23,8 & 32 & 24,6 & 10 & 7,7 & 41 & 31,5 \\
\hline Tabaco & 0 & 0,0 & 6 & 14,3 & 13 & 30,9 & 8 & 19,0 & 10 & 23,8 & 5 & 11,9 \\
\hline Maconha & 0 & 0,0 & 0 & 0,0 & 1 & 7,7 & 7 & 53,8 & 3 & 23,1 & 2 & 15,4 \\
\hline Cocaína & 0 & 0,0 & 0 & 0,0 & 0 & 0,0 & 1 & 50,0 & 1 & 50,0 & 0 & 0,0 \\
\hline Anfetaminas & 0 & 0,0 & 0 & 0,0 & 2 & 16,7 & 4 & 33,3 & 4 & 33,3 & 2 & 16,7 \\
\hline Inalantes & 0 & 0,0 & 0 & 0,0 & 3 & 11,1 & 12 & 44,4 & 7 & 16,6 & 5 & 18,5 \\
\hline Ansiolíticos & 0 & 0,0 & 1 & 7,1 & 0 & 0,0 & 2 & 14,3 & 8 & 57,1 & 3 & 21,4 \\
\hline
\end{tabular}

De acordo com a Tabela 5, a maioria dos pesquisados costumava beber em bares, danceterias e boates $(48,6 \%)$, casa de amigos $(16,9 \%)$ e em sua própria casa $(11,5 \%)$. A cerveja e o chope foram as bebidas mais consumidas pelos universitários (42,6\%), seguidas do vinho $(11,5 \%)$ e da vodka (6,1\%). A maioria dos universitários costumava beber na companhia de amigos $(57,4 \%)$ e de familiares (8,1\%). Dos universitários entrevistados, 10,8\% mencionaram que após o uso de bebidas alcoólicas faltaram à faculdade, 6,8\% dirigiram, 6,1\% brigaram após beber e 4,1\% sofreram acidentes. Ao serem questionados sobre o membro da família que bebe demais, $15,5 \%$ responderam o pai e $16,9 \%$ os tios. O uso de álcool até a embriaguez foi relatado por $50 \%$ dos universitários.

Tabela 5. Distribuição dos estudantes do curso de Farmácia e Bioquímica da Universidade Federal do Espírito Santo, segundo características do consumo de bebidas alcoólicas.

\begin{tabular}{|c|c|c|}
\hline Variáveis & $\mathrm{n}$ & $\%$ \\
\hline \multicolumn{3}{|l|}{ Bebidas consumidas } \\
\hline Cerveja & 63 & 42,6 \\
\hline Vinho & 17 & 11,5 \\
\hline Vodka & 9 & 6,1 \\
\hline Sidra & 2 & 1,4 \\
\hline Outras & 4 & 2,8 \\
\hline \multicolumn{3}{|l|}{ Doses consumidas } \\
\hline 1 a 2 doses & 65 & 42,6 \\
\hline 3 a 4 doses & 20 & 13,6 \\
\hline 5 a 6 doses & 18 & 12,2 \\
\hline 7 a 9 doses & 2 & 1,4 \\
\hline 10 ou mais doses & 2 & 1,4 \\
\hline \multicolumn{3}{|l|}{ Local de uso } \\
\hline Bares/danceterias/boates & 72 & 48,6 \\
\hline Casa de amigos & 25 & 16,9 \\
\hline Em casa & 17 & 11,5 \\
\hline \multicolumn{3}{|c|}{ Pessoas com as quais costumam beber } \\
\hline Amigos & 85 & 57,4 \\
\hline Familiares & 12 & 8,1 \\
\hline Sozinho & 2 & 1,4 \\
\hline Outros & 2 & 1,4 \\
\hline \multicolumn{3}{|l|}{ Eventos ocorridos após beber } \\
\hline Faltou à faculdade & 16 & 10,8 \\
\hline Dirigiu & 10 & 6,8 \\
\hline Brigou & 9 & 6,1 \\
\hline Sofreu acidente & 6 & 4,1 \\
\hline Nenhum & 107 & 72,3 \\
\hline
\end{tabular}

Entre os alunos que fizeram uso de inalantes, as substâncias utilizadas por último foram: lança-perfume (52,6\%), loló (22,2\%), benzina $(14,8 \%)$ e esmalte e tíner $(3,7 \%)$. Bares, danceterias e boates e a própria casa foram os locais de experimentação mais citados, com 51,9\% e 14,8\%, respectivamente. As faixas etárias mais encontradas para a experimentação de inalantes foram: 13 a 15 anos (11,1\%), 16 a 18 anos $(44,4 \%)$ e acima dos 18 anos (25,9\%). Ao serem questionados sobre a forma de obtenção dos inalantes, 51,9\% dos universitários responderam com amigos, 18,5\% compraram e 7,4\% em casa.

Questionados se alguma vez já injetaram alguma substância, 1,4\% afirmou o uso. Por outro lado, 11,5\% disseram conhecer alguém que já injetou alguma substância. O uso de anabolizantes foi relatado por 0,7\% dos entrevistados.

\section{DISCUSSÃO}

O presente estudo mostra que, entre as substâncias psicoativas, o uso das substâncias lícitas encontra-se em primeiro lugar. O Cebrid demonstrou, em seu I Levantamento Domiciliar sobre o Uso de Drogas Psicotrópicas, que 68,7\% já fizeram uso na vida de álcool e 41,1\% uso de tabaco². Entre os universitários, o uso na vida de álcool e tabaco apresenta percentuais pouco maiores e menores, respectivamente, que na população em geral, sendo encontrados $87,7 \%$ para o álcool e 30,7\% para o tabaco'.

O álcool e o tabaco apesar de serem drogas não são vistas como tal pela sociedade, que acaba estimulando o seu uso por meio de propagandas e do fácil acesso. O fácil acesso a estas substâncias é demonstrado pelo fato de que $80,3 \%$ dos universitários experimentaram álcool com 18 anos ou menos', enquanto para o tabaco 89,4\% fizeram o uso inicial com 19 anos ou menos ${ }^{8}$. Entre os estudantes de Farmácia, já foi demonstrado que o uso inicial de álcool ocorre substancialmente antes da entrada na faculdade e com menos de 21 anos ${ }^{9}$.

A busca pela facilitação da interação social é a principal expectativa do universitário ao consumir bebida alcoólica, seguida pela expectativa de redução da tensão psicológi$\mathrm{ca}^{10}$. Com esta busca, muitos acabam apresentando conseqüências após o uso de bebidas alcoólicas. No presente estudo, o consumo de bebidas alcoólicas previamente ao ato de dirigir o veículo foi a segunda atitude, relacionada ao uso de álcool, mais encontrada entre os estudantes de Farmácia. Andrade et al."1, em seu estudo com acadêmicos de medicina, demonstrou que quase metade $(44,6 \%)$ dos jovens ingeriu bebidas alcoólicas previamente à condução de veículo automotor e 20\% das jovens já o fizeram também. A diferença encontrada pode ser explicada porque alunos de Medicina tendem a ter poder aquisitivo relativamente 
alto e o carro como principal meio de transporte, fato nem sempre observado pelos acadêmicos de Farmácia.

O consumo de tabaco é considerado pela OMS uma das principais causas de morbimortalidade no Ocidente ${ }^{12}$. Entre a população brasileira, o uso na vida de tabaco é de $41,1 \%{ }^{2}$, já entre os universitários, o uso na vida desta substância não é muito diferente da população em geral, sendo encontrado $44 \%{ }^{13}, 30,7 \% 1{ }^{1}, 33 \%{ }^{4}$ e $38 \%{ }^{14}$. Neste estudo, a quantidade de cigarros consumidos por dia não foi significativa, entretanto, observa-se que, entre os universitários, a maioria consome menos de dez cigarros por dia, ${ }^{8,15}$. Não obstante o elevado uso na vida de tabaco, observa-se diminuição ao longo dos $a_{n o s}{ }^{16}$ e grande consciência entre os universitários de que seu uso é prejudicial à saúde ${ }^{15}$. Por meio deste estudo, foi possível observar "uso freqüente" e "uso pesado" relativamente baixo, demonstrando que o tabaco, não obstante ser droga lícita, como o álcool, gera grande preocupação da população sobre seus malefícios, danos amplamente divulgados pelo governo, por meio de suas campanhas contra o tabagismo.

Ainda que o consumo de substâncias psicoativas ocorra com maior prevalência entre as substâncias lícitas, as ilícitas, em especial o êxtase, são apontadas como as drogas mais perigosas pelos universitários ${ }^{17}$. No presente estudo, observou-se que os inalantes apresentaram maior uso na vida (17,6\%), dados de acordo com os encontrados em outros estudos ${ }^{1,14,18}$. No trabalho de Canuto et al.! ${ }^{18}$, o lançaperfume foi a substância mais utilizada pelos universitários, ao contrário da população em geral'19. Esta diferença pode ser explicada pelos fatores sociais de cada população: os universitários tendem a pertencer à classe social com maior poder aquisitivo, enquanto os outros inalantes estão associados às classes de rendas mais baixas'.

Conforme mostrado em outros estudos, ${ }^{4,18}$, os inalantes apresentaram maior uso na vida, enquanto a maconha apresentou maior uso no mês. Já, os dados internacionais demonstram porcentagens muito maiores de uso na vida destas substâncias ${ }^{17,18}$, como o estudo realizado com acadêmicos de Farmácia da Universidade de Praga, onde foi observado que $14,7 \%$ já fizeram uso na vida de maconha, enquanto $7,7 \%$ usaram mais de 20 vezes $^{20}$. O uso da maconha no Brasil não foi tão alto quanto em estudos internacionais, em virtude de essa substância ter seu uso regulamentado em alguns países europeus. Assim, apesar de o uso de maconha encontrado não ter sido tão alto quanto em estudos internacionais, seu uso por universitários merece atenção, uma vez que a maioria dos universitários é favorável ao seu uso e o justifica como fuga de problemas ${ }^{21}$.

Entre as outras substâncias ilícitas, as que apresentaram maior uso na vida foram: ansiolíticos (9,5\%) e anfetaminas $(8,1 \%)$. No estudo de Lucas et al.' com universitários da área da saúde, o uso na vida de anfetaminas foi de 9,2\%, enquanto o uso na vida de ansiolíticos foi de 9,2\%. Este fato de- monstra que este alto índice não é somente observado em estudantes de Farmácia, mas sim em todos os universitários de ciências da saúde. O uso destas substâncias é preocupante nesta população, especialmente no sexo feminino, pois demonstra a situação atual da sociedade, que estimula a busca por resultados imediatos e, algumas vezes, prejudiciais à saúde.

Como já relatado por Canuto et al.18, encontrar amostra representativa de uso na vida de substâncias injetáveis é extremamente difícil, nesta pesquisa somente 1,4\% dos pesquisados já fez, entretanto, 11,5\% conhecem alguém que o faz.

O uso experimental da maioria das substâncias psicoativas neste estudo foi feito antes dos 18 anos, sugerindo que o início de seu uso ocorre antes do ingresso na faculdade ${ }^{1,4,9}$. O que demonstra a importância de investimentos na prevenção do uso de substâncias psicoativas entre adolescentes, por meio da educação em saúde e do envolvimento deles nesta problemática.

\section{CONCLUSÕES}

O presente estudo mostra que os estudantes de Farmácia da Ufes apresentam perfil semelhante a outros universitários, o que evidencia o quanto a temática é relevante. Observa-se, ainda, uso inquietante, principalmente do álcool e do tabaco, que são substâncias amplamente difundidas e aceitas pela sociedade. Assim, o uso dessas substâncias é fator preocupante, uma vez que geram danos não só ao organismo do estudante, mas também a toda a sociedade.

Enfim, a escassez de estudos específicos entre estudantes de Farmácia, em especial nacionais 5,9,15,22, dificultou a análise dos dados revelando a necessidade de outras pesquisas com esta população. Todavia, este estudo contribuiu para despertar a comunidade acadêmica para relevância de a temática substância psicoativa ser abordada nos currículos universitários, em especial naqueles envolvendo estudantes da área da saúde, por causa de sua responsabilidade futura em detectar e auxiliar no tratamento do uso abusivo de substâncias psicoativas.

Conflito de interesses: Financiamento. Fundação de Apoio a Pesquisa do Espírito Santo (Fapes). Processo oo 35386452/2006

\section{AGRADECIMENTOS}

Ao Núcleo de Estudos sobre Álcool e outras Drogas pela oportunidade de realizar a pesquisa. À Fundação de Apoio à Pesquisa do Espírito Santo (Fapes) pelo suporte financeiro. A Denis Soprani Pereira, Paula Silva Mardegan e Renata Frossad Teixeira pelo auxílio na coleta de dados. 


\section{REFERÊNCIAS}

1. Lucas ACS, et al. Uso de psicotrópicos entre universitários da área da saúde da Universidade Federal do Amazonas, Brasil. Cad Saude Publica. 2006;22(3) [acesso em 2007 Mar 3]; Disponível em: http://www.scielo.br.

2. Carlini EA, et al. I Levantamento Domiciliar sobre o uso de Drogas Psicotrópicas no Brasil: estudo envolvendo as 107 maiores cidades do país - 2001. São Paulo: Centro Brasileiro de Informações sobre Drogas Psicotrópicas (Cebrid) - Departamento de Psicobiologia da Escola Paulista de Medicina, 2002.

3. Diemeff LA, Baer JS, Kivlahan DR, Marlatt GA. Alcoolismo entre estudantes universitários: uma abordagem de redução de danos. São Paulo: Unesp; 2001.

4. Kerr-Corrêa F, Andrade AG, Bassit AZ, Boccuto NMV. Uso de álcool e drogas por estudantes de medicina da Unesp. Rev Bras Psiquiatr 1999;21(2) [acesso em 2007 Mar 3]. Disponível em: http://www.scielo.br

5. Busto U, Knight K, Janecek E, Isaac P, Parker K. A problem-based learnig course for pharmacy studentes on alcohol and psychoactive abuse disordes. Am J Pharm Educ. 1994;8.

6. Smart RG, et al. A methodology for students drug-use surveys. Geneva: World Healh Organization; 1980.

7. SPSS. Statistical Package Social Science - SPSS for Windows (versão 2005). Chicago: SPSS Inc.; 2005.

8. Andrade APA, Bernardo ACC, Viegas CAA, Ferreira DBL, Gomes TC, Sales MR. Prevalência e características do tabagismo em jovens da Universidade de Brasília. J Bras Pneumol. 2006;32(1):23-8.

9. Murawski MM, Juergens JP. A problem-based learnig course for pharmacy studentes on alcohol and psychoactive abuse disordes. Am J Pharm Educ. 1994;8.

10. Mora-Rios J, Natera G. Expectativas, consumo de alcohol y problemas asociados em Estudiantes universitários de la ciudad de México. Salud Publica Mex. 2001;43(2):89-96.

11. Andrade SM, Soares DA, Braga GP, Moreira JH, Botelho FMN. Comportamentos de risco para acidentes de trânsito: um inquérito entre estudantes de medicina a região sul do Brasil. Rev Assoc Med Bras. 2003;49(4):439-44.
12. Precioso J. Quando e porquê começam os estudantes universitários a fumar: implicações para a prevenção. Análise Psicológica. 2004;22(3) [acesso em 2007 Jun 17]. Disponível em: http://www.scielo.br.

13. Stempliuk VA, Barroso LB, Andrade AG, Nicastri S, Malbergier A. Comparative study of drug use among undergraduate students at the University of São Paulo- São Paulo campus in 1996 and 2001. Rev Bras Psiquiatr. 2005;27(3) [acesso em 2007 May 12]. Disponível em: http://www.scielo.br.

14. Fiorini JE, et al. Use of licit and illicit drugs at the University of Alfenas Cad. Saúde Publica. 2006;22(3) [acesso em 2007 Mar 15]. Disponível em: http://www.scielo.br.

15. Prat-Marin A, et al. Epidemiología del tabaquismo en los estudiantes de ciências de la salud. Rev Saúde Pública. 1994;28(2) [acesso em 2007 May 7]. Disponível em: http://www. scielo.br.

16. Menezes A, et al. Evolução temporal do tabagismo em estudantes de medicina, 1986, 1991, 1996. Rev Saude Publica. 2001;35(2):165-9.

17. Zárate M, et al. Prácticas de consumo de tabaco y otras drogas em estudiantes de ciências de la salud de uma universidad privada de Lima, Perú. Investigación y Educación em Enfermería. 2006;24(2):72-81.

18. Canuto MHA, Roberto AF, Guimarães EMB. Uso e abuso de drogas ilícitas por jovens do $1^{0}$ ano da Universidade Federal de Goiás. Rev Paul Pediatria. 2006;24(2):135-42.

19. Galduróz JCF, Noto AN, Carlini EA. IV Levantamento sobre o uso de Drogas entre Estudantes de Primeiro e Segundo Graus em 10 Capitais Brasileiras. São Paulo: Centro Brasileiro de Informações sobre Drogas Psicotrópicas (Cebrid) - Departamento de Psicobiologia da Escola Paulista de Medicina; 1997.

20. Kavalírová A, Visnovský P. Use of marijuana in pharmacy students (2000-2005). Biomed Pap Med Fac Univ Palacky Olomouc Czech Repub. 2005;149(2):477-80.

21. Coutinho MPL, Araújo LF, Gontiès B. Uso da maconha e suas representações sociais: estudo comparativo entre universitários. Psicologia em Estudo Pública. 2004; 9(3) [acesso em 2007 may 23]. Disponível em: http://www.scielo.br.

22. McAuley JW, Akers AL, Mott DA. Analysis of longitudinal pharmacy student alcohol and other druf use survey data. Am J Pharm Educ. 2001;65. 